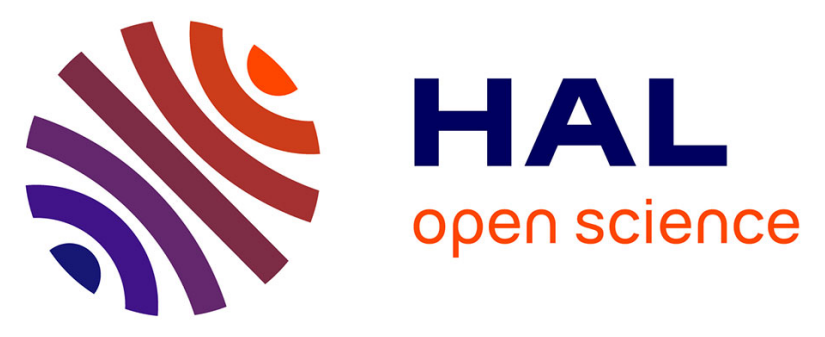

\title{
Influence of vibrational entropy on the concentrations of oxygen interstitial clusters and uranium vacancies in non-stoichiometric UO_2
}

Aurélien Soulié, Fabien Bruneval, Mihai-Cosmin Marinica, Samuel Murphy, Jean-Paul Crocombette

\section{To cite this version:}

Aurélien Soulié, Fabien Bruneval, Mihai-Cosmin Marinica, Samuel Murphy, Jean-Paul Crocombette. Influence of vibrational entropy on the concentrations of oxygen interstitial clusters and uranium vacancies in non-stoichiometric UO_2. Physical Review Materials, 2018, 2, pp.083607. 10.1103/PhysRevMaterials.2.083607 . cea-02340007

\section{HAL Id: cea-02340007 https://hal-cea.archives-ouvertes.fr/cea-02340007}

Submitted on 17 Mar 2020

HAL is a multi-disciplinary open access archive for the deposit and dissemination of scientific research documents, whether they are published or not. The documents may come from teaching and research institutions in France or abroad, or from public or private research centers.
L'archive ouverte pluridisciplinaire HAL, est destinée au dépôt et à la diffusion de documents scientifiques de niveau recherche, publiés ou non, émanant des établissements d'enseignement et de recherche français ou étrangers, des laboratoires publics ou privés. 


\title{
Influence of vibrational entropy on the concentrations of oxygen interstitial clusters and uranium vacancies in non-stoichiometric $\mathrm{UO}_{2}$
}

\author{
Aurélien Soulié(a) $^{(a)}$ Fabien Bruneval ${ }^{(a)}$, Mihai-Cosmin Marinica ${ }^{(a)}$, Samuel Murphy ${ }^{(b)}$, Jean-Paul \\ Crocombette $^{\left.(\mathrm{a}),,^{*}\right)}$
}

(a): DEN, Service de Recherches de Métallurgie Physique, CEA, Université Paris-Saclay, F-91191 Gif-surYvette, France

(b) : Department of Engineering, Lancaster University, Bailrigg, Lancaster, LA1 4YW, UK

\begin{abstract}
We combine density functional theory (DFT) formation energies and empirical potential calculations of vibrational free energies to calculate the free energies of formation of point defects and clusters of oxygen interstitials, and use a dilute defect model to calculate the concentrations of defects as a function of temperature and composition. We find that at high temperature oxygen interstitials are dominant, either in isolated form or in clusters depending on the deviation from stoichiometry. At temperatures lower than $1300 \mathrm{~K}$ we predict uranium vacancies to be dominant in the stoichiometric material. The disorder in $\mathrm{UO}_{2}$ therefore changes from Schottky to Frenkel type with increasing temperature. Uranium vacancies remain dominant up to deviations form stoichiometry as large as 0.045 at $800 \mathrm{~K}$. Moreover, the concentration of uranium vacancies proves to be non-monotonous as a function of composition. These results are consistent with some experimental data on the evolution with stoichiometry of lattice constant, diffusion coefficients of uranium, positron lifetime and dilatometry measurements.
\end{abstract}




\section{Introduction}

Uranium oxide is the most widely used nuclear fuel. As such it has been studied extensively both experimentally and numerically. This material exhibits a large domain of composition around its nominal $\left(\mathrm{UO}_{2}\right)$ stoichiometry. Deviations from stoichiometry are accommodated by the inclusion of atomic scale defects in the crystalline fluorite structure. The historical defect model $[1,2]$, dealing only with point defects, assumes that the intrinsic disorder is of the oxygen Frenkel type, i.e. that isolated oxygen vacancies and interstitials are the dominant defects in hypo and hyper-stoichiometric material respectively. This picture was further complicated by the observation of clusters of oxygen interstitials in $\mathrm{UO}_{2+x}[3]$. However, there is a general consensus on the fact that deviations from stoichiometry in $\mathrm{UO}_{2}$ are accommodated by oxygen defects. Uranium defects while predicted to exist in the material are considered to be minority defects. The main disorder is always explicitly[4, 5] or implicitly[6] assumed to be of oxygen Frenkel type.

Many atomistic calculations of the formation energies of defects exist in literature. The earliest Density Functional Theory (DFT) values date back twenty years [7] with many more published since [8-20]. In general, the calculated formation energies appear consistent with experimentally observed defect concentrations. However, when these values are integrated into a dilute defect model (for instance through a Brouwer diagram), they always predict that uranium vacancies are the most abundant defect in the hyperstoichiometric $\mathrm{UO}_{2}$ material[$[8,21]$. Calculations, therefore, point to the accommodation of hyperstoichiometry by uranium vacancies, which is direct contradiction of the dominance of oxygen interstitials in $\mathrm{UO}_{2+x}$. Atomic scale calculations, therefore, face what is widely referred to as the uranium vacancy problem. This shortcoming was observed in early DFT calculations of defect energetics in $\mathrm{UO}_{2}$ and has resisted the many subsequent improvements of the calculations (increase of the box sizes, introduction of a DFT+U correction, spin-orbit coupling, etc.).

Studies on other materials have shown that the inclusion of vibrational entropy can deeply affect the relative stability of defects in a material[22-26]. Unfortunately in the $\mathrm{UO}_{2}$ case, such calculations are impractical at the DFT level because of the tremendous computational burden they represent.

In a recent breakthrough Cooper et al [27] combined vibrational calculations performed with empirical potentials with DFT energies to estimate the free energies of formation of defects in $\mathrm{UO}_{2}$. They showed that because of differences in the vibrational entropies, the uranium vacancies are destabilized at high temperature (larger than about $1200 \mathrm{~K}$ ) compared to isolated oxygen interstitial defects. Unfortunately, 
their study dealt only with point defects and did not consider clusters of oxygen interstitials that are known to prevail at large deviations from stoichiometry. Therefore they were unable to discuss large deviations from stoichiometry and the respective stability of oxygen clusters and uranium vacancies. In the present study, we use the same combination of ab initio energies and vibrational entropies calculated with an empirical potential that enables the description of such clusters. Integrating the free energies of formation of point defects and clusters in a dilute defect model, we are able to predict the concentration of all these defects as a function of stoichiometry. At high temperatures or large deviations from stoichiometry oxygen defects are shown to be dominant. We thus confirm that the uranium vacancy problem disappears when vibrational contributions are included in the calculation of the free energies of formation. More importantly our results point to a complex picture of the hyperstoichiometry in $\mathrm{UO}_{2+x}$. Isolated oxygen interstitials, clusters of these and uranium vacancies are shown to dominate in three different regimes of compositions and temperature. Moreover, the concentration of uranium vacancies proves to be non-monotonous as a function of composition. These unexpected results are proved to be consistent with some available experimental data.

The next part of the paper presents the technicalities of our work, for the $a b$ initio and the harmonic calculations and the dilute defect model used to derive the concentrations of defects. The results are then presented and discussed.

\section{Methodology}

\section{DFT formation energies}

We use the formation energies given in our previous publication [21] to which the reader is referred for technical details. In summary, energies were calculated within the DFT theory using the VASP code[28] in the Projector Augmented Wave framework using a GGA+U functional [29] with a matrix control scheme[30, 31] to avoid the possible occurrence of local minima. All calculations were done in $2 \times 2 \times 2$ supercells of the conventional unit cell which contains 96 atoms in the perfect crystal.

One obtains the formation energies of the defects from the energy of the simulation boxes with the following formula:

$$
\Delta \mathrm{E}_{\mathrm{f}}^{\mathrm{m}, \mathrm{n}, \mathrm{q}}\left(\mu_{\mathrm{e}}\right)=\mathrm{E}\left(\mathrm{U}_{32+\mathrm{m}} \mathrm{O}_{64+\mathrm{n}}, q\right)-\frac{32+\mathrm{m}}{32} \mathrm{E}\left(\mathrm{U}_{32} \mathrm{O}_{64}\right)-\frac{\mathrm{n}-2 \mathrm{~m}}{2} \mathrm{E}\left(\mathrm{O}_{2}\right)+\mathrm{q}\left(\varepsilon_{\mathrm{VBM}}+\mu_{\mathrm{e}}\right)+\Delta \mathrm{E}_{\text {Madelung }}
$$


In this formula, $E\left(U_{32} O_{64}\right)$ is the energy of the perfect supercell and $E\left(U_{32+m} O_{64+n}, q\right)$ is the energy of the box containing a defect with $m$ uranium and $n$ oxygen added or removed atoms and charge $q . \mu_{e}$ is the electron chemical potential and $\varepsilon_{\mathrm{VBM}}$ is the energy at the top of the valence band. Finally, $E\left(\mathrm{O}_{2}\right)$ is the energy of the oxygen molecule in its triplet ground state.

We have considered the following defects :

-oxygen vacancies $V_{O}$, uranium vacancies $V_{U}$, oxygen mono interstitials $I_{O}$, and three types of oxygen interstitial clusters : $I_{X}^{2}, I_{X}^{4}$ and $I_{C}^{5}$ made built from 2, 4 and 5 oxygen interstitials respectively. For the last defect charge states were limited from 0 to -4 as higher charge states prove unstable. We also considered the hole and electron polarons explicitly by introducing a $\mathrm{U}^{5+}$ or $\mathrm{U}^{3+}$ ion in the supercell. The values of the energies obtained with equation (1) are given in appendix A of our previous paper[21].

\section{SMTB-Q vibrational free energies}

We calculate the vibrational entropies and the corresponding contributions to the free energies of the defects using the SMTB-Q (Second Moment Tight Binding with charge eQuilibration) empirical potential[32]. This potential includes a $2^{\text {nd }}$ moment tight-binding formalism for the $\mathrm{U}-\mathrm{O}$ bonding and a Buckingham term for the $\mathrm{O}-\mathrm{O}$ bonding. The charges on the atoms are equilibrated with the Electronegativity Equalization Method (EEM) [33]. The parametrization of this potential is the same as in our study on dislocations and plasticity in $\mathrm{UO}_{2}$ [34]. The ability of the SMTB-Q potential to reproduce the properties of bulk $\mathrm{UO}_{2}$ is discussed in this previous work. The SMTB-Q potential reproduces accurately the reaction energies for intrinsic Frenkel and Schottky defect processes from experiment and DFT, see table 1. The binding energies of oxygen interstitial clusters are given per oxygen interstitial in the cluster to allow direct comparison of the relative stability of the various clusters. All the defects are taken in their neutral state for this comparison. The last column gives the energy gained when 2 isolated oxygen interstitials are changed into an additional elementary cell of $\mathrm{UO}_{2}$ containing a uranium vacancy. The binding energies of DFT and empirical potentials are rather close and point qualitatively to the same trends: energy is gained when isolated interstitials are clustered; $I_{X}^{2}$ are the least stable clusters; the maximum energy gain is obtained when forming a uranium vacancy at the expense of oxygen interstitials. One difference appears for the relative stability of $I_{X}^{4}$ and $I_{C}^{5}$ (i.e. cuboctahedral) clusters. SMTB-Q predicts $I_{C}^{5}$ to be the most stable cluster while DFT calculations predict the opposite consistently with former comparable DFT calculations [13]. Note, however, that the $I_{X}^{4}$ cluster has not been observed 
experimentally while the cuboctahedral cluster is the building block of the $\mathrm{U}_{4} \mathrm{O}_{9}$ crystalline structure. We believe there may be an issue in the DFT calculations possibly associated with the limited supercell size, as evidenced by Burr and Cooper [20] for the tri-vacancy defects in the same material.

Table 1: Defect energies calculated with the SMTB-Q potential, comparison with experimental values, DFT, and CRG results.

\begin{tabular}{lllll}
\hline & \multicolumn{2}{l}{ Reaction energies $(\mathrm{eV})$} & \multicolumn{2}{l}{ Migration energies $(\mathrm{eV})$} \\
\hline Defect & $\mathrm{FP}_{\mathrm{O}}$ & Schottky Trio & $\mathrm{O}_{\mathrm{I}}$ & $\mathrm{V}_{\mathrm{O}}$ \\
SMTB-Q & 4.25 & 5.23 & 1.02 & 0.36 \\
CRG[35] & 5.73 & 10.64 & & \\
Exp. [4, 5, 36-40] & $3.0-4.6$ & $6.0-7.0$ & $0.67-1.3$ & $0.38-0.6$ \\
DFT [12, 18,41] & $2.6-4.2$ & $3.9-6.4$ & 1.14 & 0.38
\end{tabular}

\begin{tabular}{|c|c|c|c|c|}
\hline Binding energies & Per oxyg & & & \\
\hline & $I_{X}^{2}$ & $I_{X}^{4}$ & $I_{C}^{5}$ & $V_{U}$ \\
\hline DFT[21] & -0.20 & -0.83 & -0.78 & -0.97 \\
\hline SMTB-Q & 0.01 & -0.63 & -0.81 & -1.64 \\
\hline
\end{tabular}

The vibrational contributions to entropy $\left(S_{v i b}^{m, n}\right)$ and free energy $\left(F_{v i b}^{m, n}\right)$ are calculated in the harmonic approximation [42]. We make use of the PHONDY (PHONon DYynamics) code developed by Marinica et al. $[24,43]$. The phonon frequencies of the system are obtained from the diagonalization of the dynamical matrix in $5 \times 5 \times 5$ perfect or defective supercells. The phonon density of states (DOS) for perfect uranium oxide, calculated in a $10 \times 10 \times 10$ supercells, is shown in Figure 1 . In this figure one also shows the neutron weighted phonons density of states which is compared to recent inelastic neutron scattering experiments. The calculated Neutron-Weighted density of states agree reasonably well with experiments, especially for the lowest frequencies which contribute the most to vibrational entropy. A variable charge potential, based on the EEM principles, cannot reproduce the insulating gap of solids and subsequently cannot distinguish between the different defect charge states. The same entropy is, therefore, attributed to all possible charges for a given defect. The SMTB-Q empirical potential is also unable to describe polaronic defects. We, therefore, neglect the vibrational entropy contribution to the free energy of the polarons. 


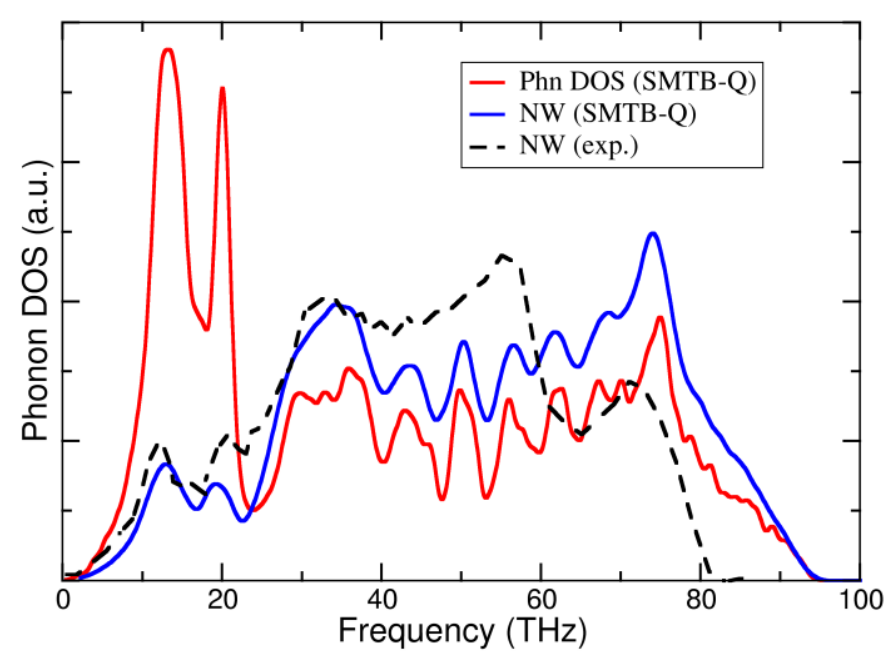

Figure 1 :Phonon density of states calculated with SMTB-Q (in red), neutron weighted phonon density of states calculated with SMTB-Q (in blue) compared to experiments extracted from Pang et al[44].

\section{Dilute defect model}

From the formation energies (Eq 1.) and vibrational free energies of the defects one can calculate the formation free energy of a given defect by the following formula:

$$
\Delta \mathrm{F}_{\mathrm{f}}^{\mathrm{m}, \mathrm{n}, \mathrm{q}}\left(\mu_{\mathrm{e}}\right)=\Delta \mathrm{E}_{\mathrm{f}}^{\mathrm{m}, \mathrm{n}, \mathrm{q}}\left(\mu_{\mathrm{e}}\right)+\Delta F_{f-v i b}^{m, n} \frac{\mathrm{n}-2 \mathrm{~m}}{2} F\left(\mathrm{O}_{2}\right)
$$

In this formula $\Delta F_{f-v i b}^{m, n}$ is the vibrational part of the free energy of formation calculated as :

$$
\Delta F_{f-v i b}^{m, n}=F_{v i b}^{m, n}-\frac{32+\mathrm{m}}{32} F_{v i b}\left(\mathrm{U}_{32} \mathrm{O}_{64}\right)
$$

(one also defines $\Delta S_{f-v i b}^{m, n}$ with an equivalent formula).

$F\left(\mathrm{O}_{2}\right)$ is the free energy of the oxygen gas beyond its internal energy term calculated by DFT (see above). It contains a pressure term, and additional terms corresponding to rotational, translational and vibrational entropies. We use the formula by Kröger[45].

The concentration of defect $X_{q}^{m, n}$ expressed in number of defects per unit cell is then :

$$
\left[X_{q}^{m, n}\right]=M^{n, p} \exp \left(-\frac{\Delta \mathrm{F}_{\mathrm{f}}^{\mathrm{m}, \mathrm{n}, \mathrm{q}}\left(\mu_{\mathrm{e}}\right)}{k_{B} T}\right)
$$

$M^{n, p}$ is the multiplicity of the defect $(m, n)$ per unit cell. This multiplicity gives the configurational entropy term and is the product of the number of sites in the unit cell and the number of equivalent ways to 
arrange the atoms to form the defect in each site. At this point the Fermi level remains to be specified. For a given pressure and temperature, it is deduced from the electro-neutrality condition which involves the concentrations of defects and the one of holes and electrons in the valence and conduction bands respectively. One finally obtains, as a function of the oxygen pressure and temperature, the concentration of each defect (including holes, electrons and polarons) and the deviation from stoichiometry $\mathrm{x}$. In the next section we present the results obtained in the hyperstoichiometric regime at various temperatures. We focus on the concentration of the various defects. We also show the variation of the the macroscopic length $(\delta \mathrm{L} / \mathrm{L})$ of a sample as a function of its stoichiometry which depends on the formation volumes of the defects as :

$$
\frac{\delta \mathrm{L}}{\mathrm{L}}=\frac{1}{3}\left(\sum_{\mathrm{X}, \mathrm{q}}\left[\mathrm{X}_{\mathrm{q}}^{\mathrm{m}, \mathrm{n}}\right] \frac{V_{\mathrm{X}}^{r \mathrm{~m}, \mathrm{n}}}{V_{\text {u.c. }}}+\sum_{V_{U, q}}\left[\mathrm{~V}_{\mathrm{U}, \mathrm{q}}\right]\right)
$$

In this equations $V_{u . c .}$ is the volume of the unit cell of $\mathrm{UO}_{2}$ and the $V_{\mathrm{X}_{\mathrm{q}}}^{\text {rel }}$ are the relaxation volumes of the defects, i.e. the variation of the supercell volume upon introduction of the defect. The calculation of these volumes is a non-trivial task as raw pressures obtained in $a b$ initio calculations are meaningless for charged cells [46]. In equation (5) the first term of the right hand side if the variation of the unit cell of the material $(\delta a / a)$ and the second term corresponds to the variation of the number of unit cell induced by the creation of uranium vacancies. Indeed we consider equilibrium between $\mathrm{UO}_{2}$ and an oxygen gas, introducing a uranium vacancy requires the creation of an additional unit cell of $\mathrm{UO}_{2}$.

\section{Results}

The values of $\Delta F_{f-v i b}^{m, n}$ for the point defects are given in Table 2 for various temperatures.

Table 2 : Vibrational contribution to the free energy of formation of defects $\Delta F_{f-v i b}^{m, n}(\mathrm{eV})$ calculated with the SMTB-Q potential (see Eq. 3).

\begin{tabular}{|c|c|c|c|c|c|c|}
\hline & $V_{O}$ & $I_{O}$ & $I_{X}^{2}$ & $I_{X}^{4}$ & $I_{C}^{5}$ & $V_{U}$ \\
\hline $400 \mathrm{~K}$ & -0.11 & -0.14 & -0.31 & -0.60 & -0.54 & -0.08 \\
\hline $700 \mathrm{~K}$ & -0.08 & -0.35 & -0.75 & -1.47 & -1.48 & -0.02 \\
\hline $1000 \mathrm{~K}$ & -0.02 & -0.60 & -1.26 & -2.48 & -2.59 & 0.08 \\
\hline $1300 \mathrm{~K}$ & 0.07 & -0.87 & -1.81 & -3.57 & -3.81 & 0.19 \\
\hline $1600 \mathrm{~K}$ & 0.17 & -1.16 & -2.40 & -4.75 & -5.14 & 0.32 \\
\hline $1900 \mathrm{~K}$ & 0.29 & -1.46 & -3.02 & -5.97 & -6.51 & 0.47 \\
\hline
\end{tabular}


Figure 2 shows the formation entropy of hyperstoichiometric defects divided by the number of oxygen interstitials they amount to. One then observes that the formation entropy of the clusters of oxygen interstitials are close to each other while the entropy of uranium vacancies is about $4 k_{B}$ per $\mathrm{O}_{1}$ smaller at all temperatures. This indicates that oxygen interstitials will be favored over uranium vacancies, especially at high temperature where the contribution of entropy to the formation free energy is important.

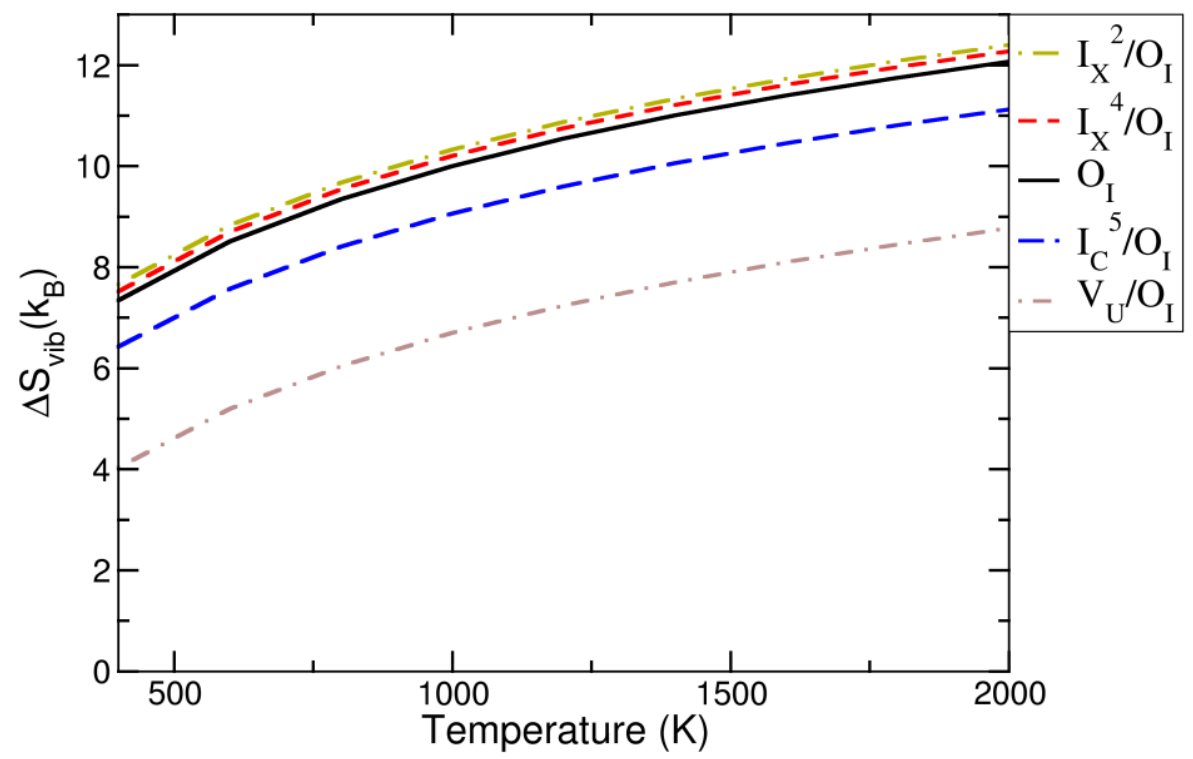

Figure 2 : Formation entropy of hyperstoichiometric defects from SMTB-Q calculations. The entropy $\Delta S_{f-v i b}^{m, n}$ are divided by the number of oxygen interstitials they contain or amount to.

We now describe the results obtained with the dilute model for the concentrations of defects. In the hypostoichiometric regime $\left(\mathrm{UO}_{2-x}\right.$, not shown), as expected, the deviation from stoichiometry is accommodated by oxygen vacancies. For the stoichiometric material (figure 3), at temperatures lower than $1300 \mathrm{~K}$ we predict uranium vacancies to be dominant. For larger temperatures, isolated interstitials take over and become dominant. In the hyperstoichiometric material, one can note that the nature of the dominant defect changes with temperature and stoichiometry (figure 3). At low temperatures (e.g. $700 \mathrm{~K})$, uranium vacancies are dominating at all deviations from stoichiometry. At these temperatures, entropy contributions are small and one finds the same results as in the calculations with DFT energies only $[16,21]$. With increasing temperature, the uranium vacancies become destabilized relative to 
oxygen interstitials. Around $1000 \mathrm{~K}$ uranium vacancies still dominate at small $\mathrm{x}$ while interstitials clusters take over above $x=0.02$. At $1300 \mathrm{~K}$ oxygen mono-interstitials and uranium vacancies appear to coexist with comparable concentrations while clusters become rapidly dominant (at $x$ larger than 0.01). Finally at $1600 \mathrm{~K}$, the hyperstoichiometry is accommodated by mono interstitials and interstitial clusters. One can note the non-monotonous evolution of the concentration of uranium vacancies: except at $700 \mathrm{~K}$ where they always dominate, their concentration increase with $x$ close to perfect stoichiometry then decreases with $\mathrm{x}$ at large hyperstoichiometry.

\section{Overstoichiometry}
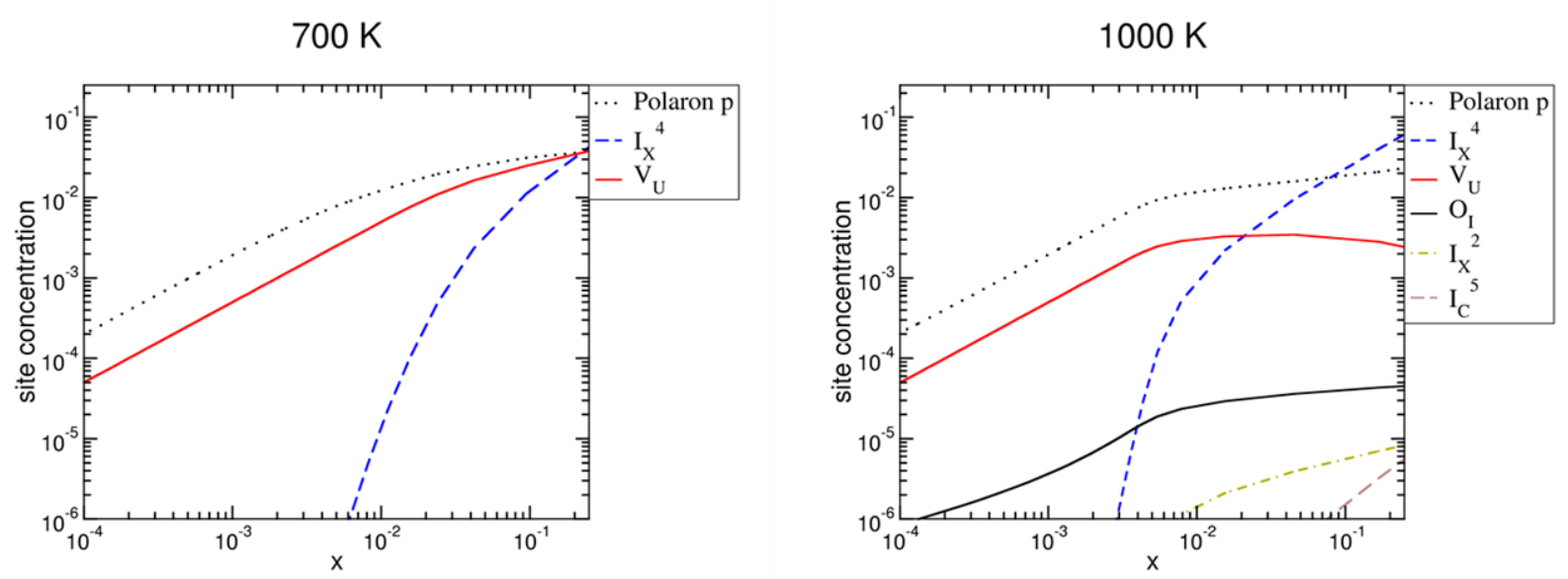

$1300 \mathrm{~K}$
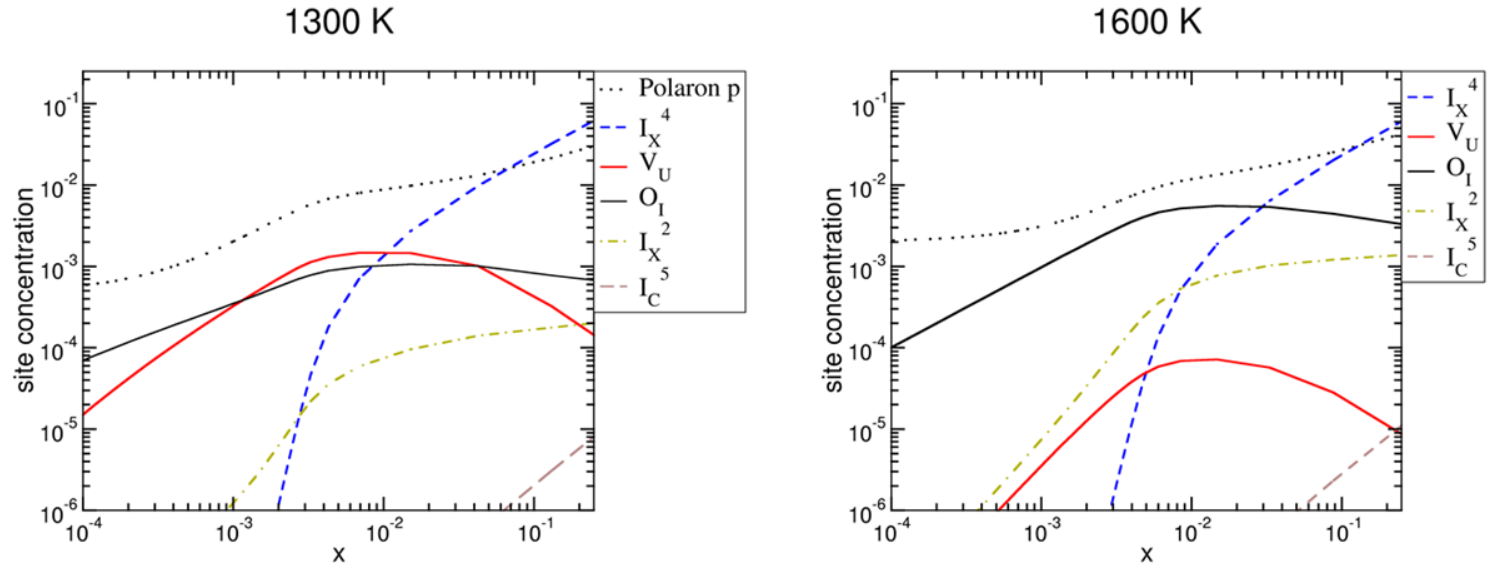

Figure 3: Concentrations of defects as a function of $\mathrm{x}$ in $\mathrm{UO}_{2+\mathrm{x}}$ for different temperatures. Note that uranium vacancies (resp. oxygen interstitials) are dominant at low (resp. high) temperatures. For clarity, we only report the total concentration for each defect where all the possible charge states have been summed up. 


\section{Discussion}

Our calculations confirm the results of Copper et al [47]. Dealing only with point defects, they have shown that including the vibrational free energies of defects stabilizes the oxygen mono-interstitials respective to the uranium vacancies. We obtain qualitatively close results when restricting our defect database of energy and entropy values to point defects only $\left(\mathrm{O}_{i}, V_{0}, V_{u}\right)$. It is interesting to note that uranium oxide has long been presented as the prototype of Frenkel disorder (with a majority of $V_{O}$ and $\mathrm{O}_{1}$ ) material. Our calculations contradict this too simple picture and suggest that the nature of the disorder in $\mathrm{UO}_{2}$ changes from Schottky (with a majority of $\mathrm{V}_{U}$ and $\mathrm{V}_{\mathrm{O}}$ ) to Frenkel type with increasing temperature.

The inclusion of clusters of oxygen interstitials allows us to explore large deviations from stoichiometry. One then obtains that clusters become dominant at large deviations from stoichiometry for all temperatures. Our results on $\mathrm{UO}_{2+x}$ can be summarized with a diagram indicating which defect is dominant as a function of temperature and stoichiometry (see Figure 4). In this figure, not all compositions are accessible. Indeed the domain of existence of $\mathrm{UO}_{2+x}$ is limited at low temperatures: a miscibility gap appears between $\mathrm{U}_{4} \mathrm{O}_{9}$ and $\mathrm{UO}_{2+\mathrm{x}}$ at temperatures lower than $1400 \mathrm{~K}$. This phase limit cannot be obtained from our modeling which describes only $\mathrm{UO}_{2+x}$. We resorted to the thermodynamical Calphad modeling of Guenau et al. [48] to draw the miscibility gap indicated by a shaded area in 4. For $x$ and $\mathrm{T}$ in this area the thermodynamically stable material is a mixture of $\mathrm{U}_{4} \mathrm{O}_{9}$ and less hyperstoichiometric $\mathrm{UO}_{2}$.

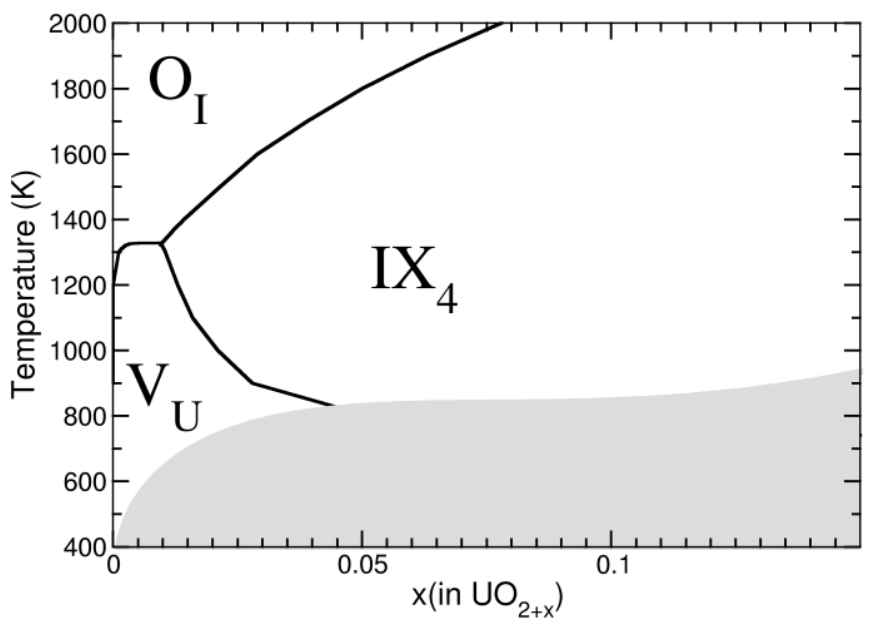

Figure 4: Dominant defect as a function of temperature and deviation from stoichiometry $(x)$ in $\cup_{2+x}$; The shaded area indicates the miscibility gap[48] between $\mathrm{UO}_{2}$ and $\mathrm{U}_{4} \mathrm{O}_{9}$ (inaccessible compositions). 
At high temperature, our results show that oxygen interstitials dominate in their isolated form at small hyperstoichiometries while clusters predominate far from perfect stoichiometry. The change from isolated to clustered interstitials takes place at increasing values of $\mathrm{x}$ with rising temperature. This is expected as high temperature favors isolated defects over clusters because of configurational entropy. Results at lower temperature are more surprising. They indicate a rather large domain of temperature and compositions where uranium vacancies dominate. Even if this domain is restricted by the limit of existence of the $\mathrm{UO}_{2+x}$ phase, we predict uranium vacancies to be the majority defects at temperatures as high as $1300 \mathrm{~K}$ and for deviations from stoichiometry as large as 0.045 around $800 \mathrm{~K}$. This prediction contradicts the widely accepted picture of the defect chemistry of $\mathrm{UO}_{2+x}$. It is indeed commonly thought that hyperstoichiometry is always accommodated by oxygen interstitials. The experimental facts underlying this assertion are the observed higher density of $\mathrm{U}_{4} \mathrm{O}_{9}$ compared to $\mathrm{UO}_{2}[49,50]$ and the neutron observations of clusters of oxygen interstitials [51]. In the latter Willis compares the absolute Bragg intensities of $\mathrm{UO}_{2}$ and $\mathrm{U}_{4} \mathrm{O}_{9}$ and states that "there is no evidence for the formation of uranium vacancies on oxidation. The uranium sublattice remains intact between $\mathrm{UO}_{2.00}$ and $\mathrm{UO}_{2.25}$, and oxidation proceeds by the incorporation of additional oxygen atoms at interstitial sites in the fluorite cell." It is worth noting however that these experiments were performed either in the two phase region of the phase diagram where one in fact has a mixture of $\mathrm{UO}_{2+x}$ and $\mathrm{U}_{4} \mathrm{O}_{9}$ phases or at high temperature. They do not contradict our suggestion of the occurrence of a region at low temperature and small deviations from stoichiometry where uranium vacancies are the dominant defects.

Moreover, there is at least one experiment clearly supporting the existence of uranium vacancies in $\mathrm{UO}_{2+\mathrm{x}}$. Desgranges et al. [52] measured the expansion of a single crystal as a function of $\mathrm{p}\left(\mathrm{O}_{2}\right)$ at $1473 \mathrm{~K}$ by dilatometry experiments and found that $\mathrm{UO}_{2+\mathrm{x}}$ first expands at low oxygen pressure (low $\mathrm{x}$ ) then contracts at large pressure (i.e. large hyperstoichiometry). A similar behavior is found at intermediate temperatures in our calculations. Figure 5 shows the elongation of $\mathrm{UO}_{2+\mathrm{x}}$ as a function of $\mathrm{x}$ at various temperatures obtained from Equation 5. This expansion then contraction is a clear sign of a large concentration of uranium vacancies in slightly hyperstoichiometric $\mathrm{UO}_{2}$. Unfortunately, Desgranges et al. results are difficult to compare quantitatively with our results as their samples contained a large amount of impurities thus blurring the $\mathrm{p}\left(\mathrm{O}_{2}\right)-x$ relationship in their experiments[53]. We believe it would be interesting to perform additional experiments of this kind to check our prediction about uranium vacancies being the dominant defect in $\mathrm{UO}_{2+x}$ at low and intermediate temperatures (below $1200 \mathrm{~K}$ ) and moderate deviations from stoichiometry. 


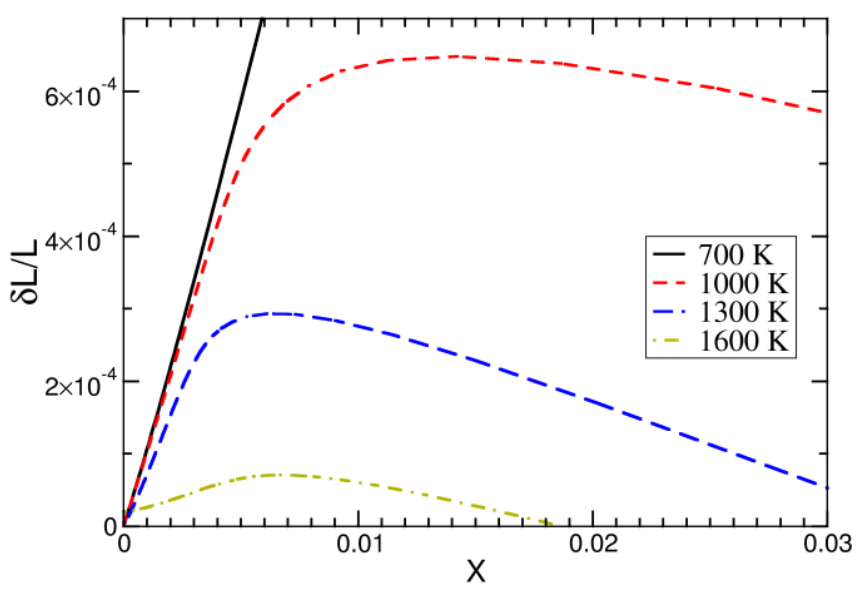

Figure 5: Elongation of $\mathrm{UO}_{2+x}$ relative to stoichiometric $\mathrm{UO}_{2}$ as a function of $x$ for different temperatures.

Another unexpected result from our simulations appears in the high temperature regime when oxygen clusters are dominant. Far from stoichiometry one can observe that concentration of uranium vacancies is decreasing. Simple arguments would suggest that the larger the deviation from stoichiometry is, the larger the concentration of uranium vacancies should be. This is in fact not the case because of the charge of these vacancies and of the evolution of the Fermi level. Hyperstoichiometric defects, such as the dominant oxygen interstitials clusters have negative to neutral charges. As their concentrations increases, more and more hole polarons appear in the material to compensate their charge. This induces a decrease of the Fermi level which is shifted towards the valence band (more precisely towards the polaronic defect level in the gap). Charge transition levels of the uranium vacancies are such that they always have their formal charge (-4) whatever the position of the Fermi level in the gap. Decreasing the Fermi level thus induces an increase of their formation energy (see equation 1 ) which in turn leads to a decrease in their concentration. Some experimental observations tend to confirm this non-monotonous evolution of the uranium vacancy concentrations.

First positron lifetime measurements carried out by Yue[54] for four well controlled compositions $(x=0.002,0.042,0.16,0.21)$ showed an increase then a decrease of the lifetime of positrons $(167,265$, $256,171 \mathrm{ps}$ ) as a function of $\mathrm{x}$. Positron lifetimes relate monotonously to the concentration of vacancies in a material. In $\mathrm{UO}_{2+\mathrm{x}}$ only uranium vacancies are significant, therefore, one can then deduce qualitatively from these experiments that the concentration of uranium vacancies increases then decreases with increasing hyperstoichiometry, as predicted in our calculations. Second, another hint of a 
possible decrease of the concentration of uranium vacancies at large $\mathrm{x}$ is given by the self-diffusion coefficients of uranium in hyperstoichiometric uranium oxide which is mediated by uranium vacancies. The measured diffusion coefficient scales as the product of the vacancy concentration by the jump frequency of each vacancy. Experimentally, one observes a general increase of the diffusion coefficient with the deviation from stoichiometry. However, at least one experiment by Hawkins et al [55] shows a final drop of the diffusion at the largest measured hyperstoichiometries for two temperatures. Assuming a constant jump frequency, such a reduction points to a final decrease of the concentrations of uranium vacancies in largely hyperstoichiometric oxide, in qualitative agreement with our calculations.

Both these experiments are thus consistent with a decrease of the concentration of uranium vacancies at large deviations from stoichiometry. They point however to a larger value of $\mathrm{x}$ for the maximum amount of uranium vacancies, somewhere around $x=0.1$ when we predict a maximum for around 0.01 . This difference may seem large, but it corresponds in the Brouwer diagram to a quite moderate change in the chemical potential of oxygen of only $0.3 \mathrm{eV}$ or equivalently one order of magnitude in the oxygen pressure.

Additional experimental confirmation of the non-monotonous evolution with stoichiometry of the concentration of uranium vacancies would validate our calculations as a whole and thus the prediction of a (temperature-stoichiometry) regime where uranium vacancies are dominant.

\section{Conclusions}

Our calculations exhibit a change in the nature of disorder in $\mathrm{UO}_{2}$ from Schottky to Frenkel type with increasing temperature. They also point to a quite complex accommodation of hyperstoichiometry in $\mathrm{UO}_{2+\mathrm{x}}$. At low deviations from stoichiometry isolated interstitials are the dominant defect. Conversely large deviations from stoichiometry are accommodated by clusters of oxygen interstitials. However, strikingly, at temperatures lower than $1300 \mathrm{~K}$ we predict uranium vacancies to be dominant up to deviations form stoichiometry as large as 0.045 at $800 \mathrm{~K}$. The actual existence of such majority uranium vacancies may be hampered by the much slower diffusivity of uranium compared to oxygen so that the true thermodynamic equilibrium may be difficult to observe for kinetic reasons. However, some experimental observations indicate that uranium vacancies may be present in non-negligible concentrations in hyperstoichiometric $\mathrm{UO}_{2}$ and that the predicted non-monotonous evolution of their concentration may be observed experimentally. We hope this simulation work will stimulate further 
experiments, possibly dilatometry or diffusion measurements, to assess the actual concentration of uranium vacancies in moderately hyperstoichiometric uranium oxide at intermediate temperatures.

\section{Acknowledgements}

Alain Chartier and Prantik Chakraborty are gratefully thanked for the phase limit appearing in figure 4.

This work was performed using HPC resources from GENCI-IDRIS and GENCI-TGCC (Grant No. 2013gen6018).

\section{References}

$1 \quad$ Hj. Matzke, Canadian Report AECL-2585 (1966).

2 A. B. Lidiard, J. Nucl Mater 19, 106 (1966).

3 B. T. M. Willis, Acta Crystallographica Section A 34, 88 (1978).

$4 \quad$ H. Matzke, J. Chem. Soc. Faraday Trans. 2, 83 (1987).

5 G. E. Murch and C. Catlow, J. Chem. Soc. Faraday Trans. 2, 83 (1987).

$6 \quad$ M. Hutchings, J. Chem. Soc., Faraday Trans. 2 83, 1083 (1987).

$7 \quad$ T. Petit, C. Lemaignan, F. Jollet, B. Bigot, and A. Pasturel, Phil. Mag. B 77, 779 (1998).

8 J. P. Crocombette, F. Jollet, L. Thien Nga, and T. Petit, Phys. Rev. B 64, 104107 (2001).

$9 \quad$ M. Freyss, T. Petit, and J. P. Crocombette, J. Nucl. Mater. 347, 44 (2005).

10 F. Gupta, G. Brillant, and A. Pasturel, Philosophical Magazine 87, 2561 (2007).

11 H. Y. Geng, Y. Chen, Y. Kaneta, and M. Kinoshita, Physical Rev. B 77, 180101(R) (2008).

12 P. Nerikar, T. Watanabe, J. S. Tulenko, S. R. Phillpot, and S. B. Sinnott, J. Nucl. Mater. 384, 61 (2009).

13 D. A. Andersson, J. Lezama, B. P. Uberuaga, C. Deo, and S. D. Conradson, Physical Rev. B 79, 024110 (2009).

14 J. G. Yu, R. Devanathan, and W. J. Weber, J. Phys.-Condes. Matter 21, 435401 (2009).

15 B. Dorado, G. Jomard, M. Freyss, and M. Bertolus, Phys. Rev. B 82, 035114 (2010). 
J.-P. Crocombette, D. Torumba, and A. Chartier, Phys. Rev. B 83, 184107 (2011).

J. P. Crocombette, Phys. Rev. B 85, 144101 (2012).

18

E. Vathonne, J. Wiktor, M. Freyss, G. Jomard, and M. Bertolus, J. Phys.-Condes. Matter 26, 325501 (2014).

19 J. Wang, R. C. Ewing, and U. Becker, Sci. Rep. 4, 4216 (2014).

20 P. A. Burr and M. W. D. Cooper, Phys. Rev. B 96, 094107 (2017).

21 F. Bruneval, M. Freyss, and J.-P. Crocombette, Physical Review Materials 2, 023801 (2018).

22 C. Freysoldt, B. Grabowski, T. Hickel, J. Neugebauer, G. Kresse, A. Janotti, and C. G. Van de Walle, Reviews of Modern Physics 86, 53 (2014).

23 D. Murali, M. Posselt, and M. Schiwarth, Phys. Rev. B 92, 18 (2015).

24 L. Proville, D. Rodney, and M.-C. Marinica, Nature Materials 11, 845 (2012).

25 K. Carling, G. Wahnstrom, T. R. Mattsson, A. E. Mattsson, N. Sandberg, and G. Grimvall, Physical Review Letters 85, 3862 (2000).

26 D. A. Terentyev, T. P. C. Klaver, P. Olsson, M. C. Marinica, F. Willaime, C. Domain, and L. Malerba, Physical Review Letters 100, 4 (2008).

27 M. W. D. Cooper, S. T. Murphy, and D. A. Andersson, J. Nucl. Mater. 504, 251 (2018).

$28 \quad$ G. Kresse and J. Furthmuller, Phys. Rev. B 54, 11169 (1996).

29 A. I. Liechtenstein, V. I. Anisimov, and J. Zaanen, Phys. Rev. B 52, R5467 (1995).

30 B. Dorado, B. Amadon, M. Freyss, and M. Bertolus, Phys. Rev. B 79, 235125 (2009).

31 F. Jollet, G. Jomard, B. Amadon, J. P. Crocombette, and D. Torumba, Phys. Rev. B 80, 235109 (2009).

32 A. Hallil, R. Tetot, F. Berthier, I. Braems, and J. Creuze, Phys. Rev. B 73, 10 (2006).

33 A. K. Rappe and W. A. Goddard, The Journal of Physical Chemistry 95, 3358 (1991).

34 A. Soulié, J.-P. Crocombette, A. Kraych, F. Garrido, G. Sattonnay, and E. Clouet, Acta Mater. 150, 248 (2018).

35 M. W. D. Cooper, M. J. D. Rushton, and R. W. Grimes, Journal of Physics Condensed Matter 26, 105401 (2014).

$36 \quad$ K. Clausen, W. Hayes, M. T. Hutchings, J. E. Macdonald, R. Osborn, and P. Schnabel, Rev. Phys. Appl. (Paris) 19, 719 (1984). 
37 D. Staicu, T. Wiss, V. V. Rondinella, J. P. Hiernaut, R. J. M. Konings, and C. Ronchi, J. Nucl. Mater. 397, 8 (2010).

$38 \quad$ J. Belle, J. Nucl. Mater. 30, 3 (1969).

39 B. Dorado, P. Garcia, G. Carlot, C. Davoisne, M. Fraczkiewicz, B. Pasquet, M. Freyss, C. Valot, G. Baldinozzi, D. Siméone, and M. Bertolus, Phys. Rev. B 83, 035126 (2011).

$40 \quad$ K. C. Kim and D. R. Olander, J. Nucl. Mater. 102, 192 (1981).

41 J. P. Crocombette, Phys. Rev. B 85, 144101 (2012).

42 A. A. Maradudin, E. W. Montroll, G. H. Weiss, and I. P. Ipatova, (Academic Press, New York, 1971), p. 130 chapter IV,

43 M. C. Marinica and F. Willaime, in Solid State Phenomena, 2007), Vol. 129, p. 67.

$44 \quad$ J. W. L. Pang, A. Chernatynskiy, B. C. Larson, W. J. L. Buyers, D. L. Abernathy, K. J. McClellan, and S. R. Phillpot, Phys. Rev. B 89, 115132 (2014).

45 F. A. Kröger, The Chemistry of imperfect crystals, vol.1 (North-Holland publishing company, Amsterdam, 1973),

46 F. Bruneval, C. Varvenne, J.-P. Crocombette, and E. Clouet, Phys. Rev. B 91, 024107 (2015).

47 M. W. D. Cooper, S. T. Murphy, and D. A. Andersson, J. Nucl. Mater. (2018).

48 C. Guéneau, M. Baichi, D. Labroche, C. Chatillon, and B. Sundman, J. Nucl. Mater. 304,161 (2002).

$49 \quad$ F. Grønvold, J. Inorganic and Nuclear Chemistry 1, 355 (1955).

50 H. R. Hoekstra, A. Santoro, and S. Siegel, Journal of Inorganic and Nuclear Chemistry 18, 166 (1961).

51 B. T. M. Willis, J. Chem. Soc., Faraday Trans. 2 83, 1073 (1987).

52 L. Desgranges, M. Gramond, C. Petot, G. Petot-Ervas, P. Ruello, and B. Saadi, Journal of the European Ceramic Society 25, 2683 (2005).

$53 \quad$ L. Desgranges (private communication).

$54 \quad$ Y. Ma, A study of point defects in UO2+x and their impact upon fuel properties https://tel.archives-ouvertes.fr/tel-01714468, PhD thesis, Université d'Aix-Marseille, 2018.

55

R. J. Hawkins and C. B. Alcock, J. Nucl. Mater. 26, 112 (1968). 